\title{
Comparitive Studies of Futuristic Optimisezed and Regular Solar Panels with Windload Effects on Solar Farms, Solar Panels at High Altitude for Free Green Solar Energy
}

\author{
P. Surendra Reddy ${ }^{1}$, K. Swapna ${ }^{1}$, G. Kiran Kumar ${ }^{2,}$ \\ ${ }^{1}$ Department of Physics, Koneru Lakshmaiah Education Foundation, Green Fields, Guntur, AP, India. \\ ${ }^{2}$ Department of Physics, Raghu Engineering College, Dakamarri, Visakhapatnam- 531162, AP, India.
}

Article History: Received: 10 November 2020; Revised 12 January 2021 Accepted: 27 January 2021; Published online: 5 April 2021

\begin{abstract}
:
Solar energy is one of the most promising green and eco-friendly renewable energy out of the wind and other non-conventional energies. In nature, we have abundance of solar energy, due to this there is a significant attention on capturing the solar energy by photovoltaic systems in recent years. Photovoltaic energy conversion is most harnessing renewable energies for different communities especially in developing countries like India. The efficiency decrease is not only due to the shadows of buildings, but also due to birds, lack of sunlight and also wind effect on solar panels which will decrease efficiency and damage the total panels or array of panels. In this work, two different analyses are carried out with changing wind velocities and angle. The aerodynamic effects on the panels were investigated by varying the orientation of panels from 0 to 180 degrees and two different wind speeds of $5 \mathrm{~m} / \mathrm{s}$ and $25 \mathrm{~m} / \mathrm{s}$. Initial angle of 0 degrees which is normal to the flow direction is solved for two different wind speeds of $5 \mathrm{~m} / \mathrm{s}$ and $25 \mathrm{~m} / \mathrm{s}$ and followed by changing the orientation of panels from 0 to 180 degree inclination to investigate the aerodynamic effects on the panels. Depending on the results obtained a futuristic panel design will be proposed which can generate optimum power and also aerodynamically effective. This current study indicates that the pressure distribution on the front face of the solar panels, which are aptly suitable to design optimized solar panel shapes.
\end{abstract}

Keywords: Solar Panel, Aero Dynamics, Urban Environment, Optimization, Free green solar energy.

\section{Introduction}

Towards the green energy approach over the past few years, commercial ground mounted solar farms installation have expanded not only in urban areas but also into agricultural and rural communities where open land is more. The farmers can give their land for producing clean and pollution free green solar energy. A solar farm is a photovoltaic power station in which solar panels are arrayed in decentralized manner supplying the power to power grid. Solar farms may be associated with both residential community-scale solar and utility-scale solar. Recently people are thinking about community scale solar, in which solar array is a large ground mount installation that occupies one or more acres. Utility scale solar is the largest scale (1 to $5 \mathrm{MW}$ ) solar installation. The main difference between community solar scale and utility solar scale is that the power generated in utility scale is not utilized at the host site. The produced $1 \mathrm{MW}$ of power in utility sector can supply power to 200 households. Even though the cost of the solar farm depends on the location and availability of sunlight, it will cost around 100 crore rupees. India installed the world's largest solar farm at Pavagada 
which was completed in 2015 with 2050 MW power generation capacity which can supply power to thousands of households.

Solar energy can potentially replace all types of renewable energy sources, but there are some major constraints for development of solar energy in India. The constraints are solar resource assessment, wind resource assessment, wind and solar farm distribution and atmospheric conditions like hurricanes, tornadoes with wind speeds up to 200 miles per hour. It is very important to know about how the solar energy system holds up in extreme wind events that may knock out the parts of the electric grid. Normally, solar farms are highly rigid to damage from stormy wind conditions. In most cases wind causes damages to solar system, failures might happen because of weakness in racking or the roof where we place the system. When wind flows across the array of the panels, it flows between the small spaces of the panels causing a lifting of the panels that leads to damage of the total solar farm. This wind flow is capable of damaging panels from their mounts, or the mounts from ground or roof. Another cause of damage is flying debris. Different types of debris that can be blown in storm can make damage to the solar array of panels.

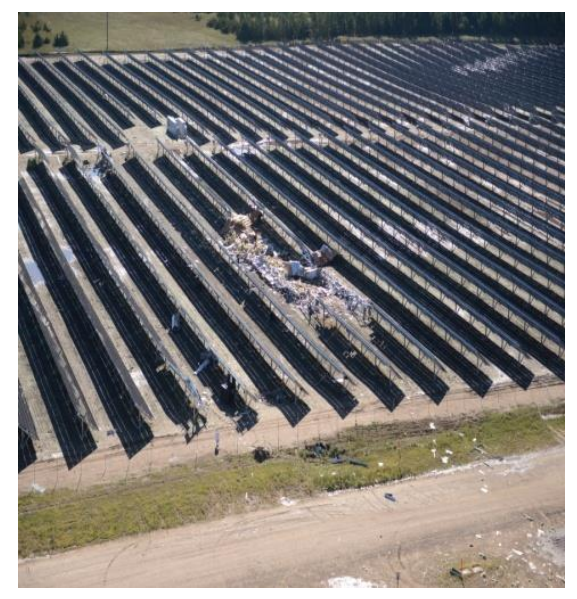

Figure 1.Camp Ripley received damage 60 acre solar field, during a big storm and tornado Sept 7, 2016.

\section{Objectives and Hypothesis}

The main objective of this work is to find a solution to the problem of ground mounted and roof mounted solar panels that are dislocated due to aerodynamic loads. The effects of forces caused due to air flow are accurately predicted in all directions by using CFD (computational Fluid Dynamics). A full scale model is used with a control domain to produce results accurately. It is hypothesized that doing CFD analysis and analyzing the data, a most accurate and reliable data can be obtained on aerodynamic forces so that uncertainties can be reduced.

Damage of solar panels: The solar panels modules and mounting structures will be affected by the wind as follows.

1. Velocity of the wind: Usually there is huge variation in wind velocities form one region to another region. Normally there is 10 to $60 \mathrm{~km} / \mathrm{hr}$ of wind speed and at the coastal regions the range will be around 60 to $90 \mathrm{~km} / \mathrm{hr}$. Similarly at the time of hurricanes the speed may rise to more than $90 \mathrm{~km} / \mathrm{hr}$. 
2. Direction of the Wind: Normally Wind direction is always changing from one direction to another direction. Since we place the solar panels at the top of the building or on the ground, wind may flow in either direction. Wind may flow in the direction of inclination of panel and sometimes opposite to that.

3. Size of the building and the presence of the protective wall: The speed of wind is varied due to increase in latitude means that there is more speed at the top. Protective wall around the building prevents the air to go into the backward position and causes lift in the upward direction.

\section{Position of the building in the environment.}

5. Inclination of the solar panels to the sides of the building i.e. either oblique or parallel.

6. Angle of tilt of the solar panels: The tilt angle of solar panels normally 12 to 18 degrees. It may change from season to season. Tilt angle is important for maximum exposure of sunlight on the panels.

7. location of the panels to the roof edges.

There are many research objectives to be considered while doing a project.

To obtain the aerodynamic loads on an arrayed ground mounted solar panels in a solar farm and the aerodynamic load effects on roof mounted solar panels. To examine the effects of pressure, velocity and turbulence in all cases. To obtain forces acting on individual solar panel to access most probable damage caused by aerodynamic load. To optimize shape of solar panel to reduce the wind forces.

\section{Methodology}

Numerical modeling program was adopted to calculate the pressure distribution on solar panels [1]. The numerical results of CFD ANSYS simulation and the pressure coefficient distribution are similar when compared to full scale measurements. [2].In the past years many people have done research on the wind effect on solar panels. Numerical studies and scaled wind tunnel test on array of PV panels were done by researchers [3, 4, 5, and 6]. Among the many parameters which influence the drag forces and lift forces, wind directions are the ones which will affect most of these forces. There are many parameters that can change the entire effect of wind on panels.

CFD ANSYS is a software tool to optimize the conditions of products and control even before making of the first prototype. Fig. 1 shows the overview of the CFD modeling. There are several steps to examine the outcomes of the effect of wind on solar panels. The initial step is problem identification and the subsequent steps are preprocessing, solving and post processing. [7]. 


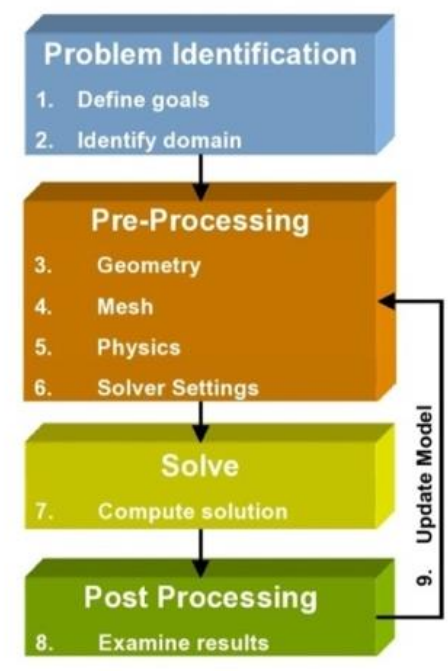

Fig.2 Overview of the CFD modeling.

\section{Results}

The computational domain is used to simulate the scenarios possible to appear in real time scenario and such following scenarios are considered. The following are the cases which are published in my previous papers $[8,9]$.

Wind direction is normal to the Panel Plane with 5 and $25 \mathrm{~m} / \mathrm{s}$ speed for rectangular and optimized panels at ground mounted.

Wind direction is reverse normal to the Panel Plane with 5 and $25 \mathrm{~m} / \mathrm{s}$ speed for rectangular and optimized panels at ground mounted.

Wind direction is normal to the Panel Plane with 5 and $25 \mathrm{~m} / \mathrm{s}$ speed for rectangular and optimized panels at roof mounted.

Wind direction is reverse normal to the Panel Plane with 5 and $25 \mathrm{~m} / \mathrm{s}$ speed for rectangular and optimized panels at roof mounted.

Table.1 Comparing the Drag forces between Rectangular and Hexagonal solar panels at wind velocity $5 \mathrm{~m} / \mathrm{s}$

\begin{tabular}{|c|c|c|c|c|}
\hline \multicolumn{5}{|c|}{$\begin{array}{l}\text { Comparing of wind effects on solar Panels mounted at } 5 \text { Storey building at Velocity } \\
\qquad 5 \mathrm{~m} / \mathrm{s}\end{array}$} \\
\hline & \multicolumn{2}{|c|}{ Rectangular Solar Panels } & \multicolumn{2}{|c|}{ Optimized Hexagonal solar panels } \\
\hline Wind Angle & $\begin{array}{c}\boldsymbol{\theta}^{\prime}=\mathbf{0}^{\circ} \\
(\text { Front Side) }\end{array}$ & $\begin{array}{c}\mathbf{\theta}=\mathbf{1 8 0}^{\circ} \\
\text { (Back Side) }\end{array}$ & $\begin{array}{c}\boldsymbol{\theta}^{\prime}=\mathbf{0}^{\mathrm{o}} \\
(\text { Front Side) }\end{array}$ & $\begin{array}{c}\begin{array}{c}\Theta=\mathbf{1 8 0}^{\circ} \\
\text { (Back Side) }\end{array} \\
\end{array}$ \\
\hline PANEL & Force $\mathbf{F}(-Z) \mathbf{N}$ & Force $\mathbf{F}(\mathbf{Z}) \mathbf{N}$ & Force $\mathbf{F}(-\mathbf{Z}) \mathbf{N}$ & Force $\mathbf{F}(\mathbf{Z}) \mathbf{N}$ \\
\hline PANEL 1 & 204.355 & 106.249 & 74.641 & 56.963 \\
\hline PANEL 2 & 191.083 & 98.393 & 80.724 & 56.921 \\
\hline PANEL 3 & 183.415 & 124.031 & 71.53 & 57.943 \\
\hline
\end{tabular}


Table.2 Comparing the Drag forces between Rectangular and Hexagonal solar panels at wind velocity $25 \mathrm{~m} / \mathrm{s}$

\begin{tabular}{|c|c|c|c|c|}
\hline \multicolumn{5}{|c|}{$\begin{array}{l}\text { Comparing of wind effects on solar Panels mounted at } 5 \text { Storey building at Velocity } \\
25 \mathrm{~m} / \mathrm{s}\end{array}$} \\
\hline & \multicolumn{2}{|c|}{ Rectangular Solar Panels } & \multicolumn{2}{|c|}{ Optimized Hexagonal solar panels } \\
\hline Wind Angle & $\begin{array}{c}\boldsymbol{\theta}=\mathbf{0}^{\circ} \\
\text { (Front Side) }\end{array}$ & $\begin{array}{c}\begin{array}{c}\theta=\mathbf{1 8 0}^{\circ} \\
\text { (Back Side) }\end{array} \\
\end{array}$ & $\begin{array}{c}\boldsymbol{\theta}=\mathbf{0}^{\mathbf{0}} \\
\text { (Front Side) }\end{array}$ & $\begin{array}{c}\boldsymbol{\theta}=\mathbf{1 8 0}^{\circ} \\
\text { (Back Side) }\end{array}$ \\
\hline PANEL NO & Force $\mathbf{F}(-Z) \mathbf{N}$ & Force $F(Z) N$ & Force $\mathbf{F}(-\mathrm{Z}) \mathbf{N}$ & Force $\mathbf{F}(\mathbf{Z}) \mathbf{N}$ \\
\hline PANEL 1 & 1048.461 & 355.516 & 505.248 & 280.93 \\
\hline PANEL 2 & 1307.698 & 720.88 & 904.305 & 502.811 \\
\hline PANEL 3 & 1137.764 & 944.868 & 469.716 & 307.695 \\
\hline
\end{tabular}

Table.3 Comparing the Lift forces between Rectangular and Hexagonal solar panels at wind velocity $5 \mathrm{~m} / \mathrm{s}$

\begin{tabular}{|c|c|c|c|c|}
\hline \multicolumn{5}{|c|}{$\begin{array}{l}\text { Comparing of wind effects on solar Panels mounted at } 5 \text { Storey building at Velocity } \\
5 \mathrm{~m} / \mathrm{s}\end{array}$} \\
\hline & \multicolumn{2}{|c|}{ Rectangular Solar Panels } & \multicolumn{2}{|c|}{ Optimized Hexagonal solar panels } \\
\hline Wind Angle & $\begin{array}{c}\boldsymbol{\theta}=\mathbf{0}^{\circ} \\
(\text { Front Side) }\end{array}$ & $\begin{array}{c}\begin{array}{c}\Theta=\mathbf{1 8 0}^{\circ} \\
(\text { Back Side) }\end{array}\end{array}$ & $\begin{array}{c}\boldsymbol{\theta}^{\prime}=\mathbf{0}^{\circ} \\
(\text { Front Side) }\end{array}$ & $\begin{array}{c}\mathbf{\theta}=\mathbf{1 8 0}^{\circ} \\
(\text { Back Side) }\end{array}$ \\
\hline PANEL NO & Force $\mathbf{F}(\mathbf{Y}) \mathbf{N}$ & Force $\mathbf{F}(\mathbf{Y}) \mathbf{N}$ & Force $\mathbf{F}(\mathbf{Y}) \mathbf{N}$ & Force $\mathbf{F}(\mathbf{Y}) \mathbf{N}$ \\
\hline PANEL 1 & 412.576 & 211.943 & 237.637 & 174.459 \\
\hline PANEL 2 & 385.285 & 196.039 & 258.404 & 174.535 \\
\hline PANEL 3 & 369.217 & 248.29 & 229.846 & 177.834 \\
\hline
\end{tabular}

Table.4 Comparing the Lift forces between Rectangular and Hexagonal solar panels at wind velocity $25 \mathrm{~m} / \mathrm{s}$

\begin{tabular}{|c|c|c|c|c|}
\hline \multicolumn{5}{|c|}{ Comparing of wind effects on solar Panels mounted at 5 Storey building at Velocity $25 \mathrm{~m} / \mathrm{s}$} \\
\hline & \multicolumn{2}{|c|}{ Rectangular Solar Panels } & \multicolumn{2}{|c|}{ Optimized Hexagonal solar panels } \\
\hline Wind Angle & $\begin{array}{c}\boldsymbol{\theta}=\mathbf{0}^{\mathbf{o}} \\
\text { (Front Side) }\end{array}$ & $\begin{array}{c}\mathbf{\theta}=\mathbf{1 8 0}^{\circ} \\
\text { (Back Side) }\end{array}$ & $\begin{array}{c}\boldsymbol{\Theta}=\mathbf{0}^{\circ} \\
\text { (Front Side) }\end{array}$ & $\begin{array}{c}\begin{array}{c}\theta=\mathbf{1 8 0}^{\circ} \\
\text { (Back Side) }\end{array}\end{array}$ \\
\hline PANEL NO & Force $\mathbf{F}(\mathbf{Y}) \mathbf{N}$ & Force $\mathbf{F}(\mathbf{Y}) \mathbf{N}$ & Force $\mathbf{F}(\mathbf{Y}) \mathbf{N}$ & Force $\mathbf{F}(\mathbf{Y}) \mathbf{N}$ \\
\hline PANEL 1 & 4817.519 & 906.98 & 1037.084 & 648.869 \\
\hline PANEL 2 & 4517.555 & 2692.649 & 895.045 & 1376.16 \\
\hline PANEL 3 & 3933.78 & 865.799 & 919.814 & 1891.854 \\
\hline
\end{tabular}

Above tables show the drag and lift forces between the rectangular solar panel and hexagonal solar panels when they are mounted at 5 storey building at velocities $5 \mathrm{~m} / \mathrm{s}$ and $25 \mathrm{~m} / \mathrm{s}$. At overall glance, the drag and lift forces are less for hexagonal solar panel due to the shape of the panels. From Table.1, drag forces on rectangular solar panel is more than $50 \%$ as compared with drag forces on the hexagonal solar panel when the wind flows with $0^{\circ}$ to the panels at $5 \mathrm{~m} / \mathrm{s}$ velocity. Furthermore the drag force is more than $50 \%$ high in case of rectangular solar panels as compared with hexagonal solar panels when wind flows $180^{\circ}$ to the panels at $5 \mathrm{~m} / \mathrm{s}$. The change of wind speed from $5 \mathrm{~m} / \mathrm{s}$ to $25 \mathrm{~m} / \mathrm{s}$ doesn't affect the outcome (see table2). But the magnitude of the drag force is more in case of wind velocity $25 \mathrm{~m} / \mathrm{s}$ which is almost 5times more as compared with velocity $5 \mathrm{~m} / \mathrm{s}$. From these we can say that optimized hexagonal solar panels will affect less amount of drag force due to the hexagonal 
shape of the panels. Due to heavy amount of drag force it can be observed that dislocation and damage of the panels occurred.

From Table.3shows that lift force is more in case of rectangular solar panels as compared with hexagonal solar panels. But in case of the $180^{\circ}$ wind flow at $25 \mathrm{~m} / \mathrm{s}$, lift force is little bit more in hexagonal solar panels which can be observed in the Table.4. This is the unpredicted behavior of the turbulence flow. Overall for the roof mounted solar panels, the drag and lift forces are less in hexagonal as compared with the rectangular solar panels. So, hexagonal solar panels are preferable for households and commercial purpose. Due to low cost, we can use maximum active area of solar radiation exposure and less amount of silicon wafer is wasted. The hexagonal solar panel can be used easily on rooftops of the building for both house using and commercial purpose. The low ratio of perimeter to area reduces the sampling bias and tessellate to form a continuous grid. Also hexagons are preferable when they are connecting the small panels into big panels. Hexagons are also better for capturing the solar radiation duo to large working area and hexagons grid will have less amount of distortion due to the curvature. Hexagonal panels have drag coefficient of 1.83 which is less than the drag coefficient of a square 1.92 due to the shape of the plane.

Table.5 Comparing the Drag forces between Rectangular and Hexagonal solar panels at wind velocity $5 \mathrm{~m} / \mathrm{s}$ mounted at ground.

\begin{tabular}{|c|c|c|c|c|c|}
\hline \multicolumn{6}{|c|}{ Comparing of wind effects on solar farm mounted at ground at Velocity of $5 \mathrm{~m} / \mathrm{s}$} \\
\hline \multicolumn{2}{|c|}{ Shape of the panel } & \multicolumn{2}{|c|}{$\begin{array}{l}\text { Rectangular Solar } \\
\text { Panels }\end{array}$} & \multicolumn{2}{|c|}{$\begin{array}{c}\text { Optimized Hexagonal solar } \\
\text { panels }\end{array}$} \\
\hline \multirow{2}{*}{ ROWS } & $\begin{array}{l}\text { Wind } \\
\text { Angle }\end{array}$ & $\begin{array}{c}\boldsymbol{\Theta}=\mathbf{0}^{\circ} \\
\text { (Front Side) }\end{array}$ & $\begin{array}{c}\begin{array}{c}\boldsymbol{\theta}=\mathbf{1 8 0}^{\circ} \\
\text { (Back Side) }\end{array}\end{array}$ & $\begin{array}{c}\boldsymbol{\theta}^{=\mathbf{0}^{\circ}} \\
\text { (Front Side) }\end{array}$ & $\begin{array}{c}\boldsymbol{\theta}=\mathbf{1 8 0}^{\circ} \\
\text { (Back Side) }\end{array}$ \\
\hline & $\begin{array}{l}\text { PANEL } \\
\text { NO }\end{array}$ & $\begin{array}{c}\text { Force } \\
\text { F }(-Z) \mathbf{N}\end{array}$ & $\begin{array}{l}\text { Force } \\
\text { F(Z) N }\end{array}$ & $\begin{array}{c}\text { Force } \\
\text { F }(-Z) \mathbf{N}\end{array}$ & $\begin{array}{l}\text { Force } \\
\text { F(Z)N }\end{array}$ \\
\hline \multirow{7}{*}{ ROW-1 } & PANEL 1 & 180.34 & 176.5408 & 57.73 & 46.33 \\
\hline & PANEL 2 & 182.17 & 171.9558 & 59.27 & 52.03 \\
\hline & PANEL 3 & 179.67 & 174.2058 & 58.59 & 53.35 \\
\hline & PANEL 4 & 182.57 & 174.6558 & 56.86 & 53.49 \\
\hline & PANEL 5 & 181.24 & 174.0558 & 60.48 & 53.5 \\
\hline & PANEL 6 & 181.37 & 175.7308 & 58.97 & 52.68 \\
\hline & PANEL 7 & 181.72 & 175.2692 & 58.47 & 46.12 \\
\hline \multirow{7}{*}{ ROW-2 } & PANEL 8 & 181.57 & 175.3442 & 22.12 & 38.45 \\
\hline & PANEL 9 & 181.86 & 178.1992 & 23.42 & 47.02 \\
\hline & PANEL 10 & 181.72 & 178.2692 & 23.68 & 47.69 \\
\hline & PANEL 11 & 181.26 & 178.4992 & 23.14 & 47.26 \\
\hline & PANEL 12 & 183.42 & 178.9192 & 23.95 & 48.12 \\
\hline & PANEL 13 & 188.82 & 176.2192 & 23.78 & 46.43 \\
\hline & PANEL 14 & 180.71 & 180.2742 & 22.95 & 39.05 \\
\hline \multirow{6}{*}{ ROW-3 } & PANEL 15 & 179.83 & 180.7142 & 21.35 & 27.49 \\
\hline & PANEL 16 & 182.44 & 180.9092 & 20.33 & 31.11 \\
\hline & PANEL 17 & 180.42 & 181.9192 & 21.1 & 31.89 \\
\hline & PANEL 18 & 178.53 & 182.8642 & 21.4 & 32.87 \\
\hline & PANEL 19 & 179.29 & 182.4842 & 20.19 & 32.99 \\
\hline & PANEL 20 & 178.53 & 182.8642 & 21.28 & 28.42 \\
\hline
\end{tabular}




\begin{tabular}{|c|c|c|c|c|c|}
\hline & PANEL 21 & 180.41 & 183.4242 & 21.28 & 28.42 \\
\hline \multirow{5}{*}{ ROW-4 } & PANEL 22 & 173.44 & 186.9092 & 20.96 & 55.6 \\
\cline { 2 - 6 } & PANEL 23 & 176.43 & 185.4142 & 21.59 & 57.35 \\
\cline { 2 - 6 } & PANEL 24 & 176.36 & 185.4492 & 21.87 & 57.29 \\
\cline { 2 - 6 } & PANEL 25 & 176.16 & 185.5492 & 21.58 & 56.82 \\
\cline { 2 - 6 } & PANEL 26 & 176.73 & 186.7642 & 20.74 & 56.57 \\
\cline { 2 - 6 } & PANEL 27 & 175.86 & 187.1992 & 21.32 & 57.18 \\
\cline { 2 - 6 } & PANEL 28 & 176.38 & 186.9392 & 21.5 & 55.61 \\
\hline
\end{tabular}

Table.6 Comparing the Drag forces between Rectangular and Hexagonal solar panels at wind velocity $25 \mathrm{~m} / \mathrm{s}$ mounted at ground

\begin{tabular}{|c|c|c|c|c|c|}
\hline \multicolumn{6}{|c|}{ Comparing of wind effects on solar farm mounted at ground at Velocity of $25 \mathrm{~m} / \mathrm{s}$} \\
\hline \multicolumn{2}{|c|}{ Shape of the panel } & \multicolumn{2}{|c|}{$\begin{array}{c}\text { Rectangular Solar } \\
\text { Panels }\end{array}$} & \multicolumn{2}{|c|}{$\begin{array}{c}\text { Optimized Hexagonal solar } \\
\text { panels }\end{array}$} \\
\hline \multirow{2}{*}{ ROWS } & $\begin{array}{l}\text { Wind } \\
\text { Angle }\end{array}$ & $\begin{array}{c}\boldsymbol{\Theta}=\mathbf{0}^{\circ} \\
\text { (Front Side) }\end{array}$ & $\begin{array}{c}\Theta=\mathbf{1 8 0}^{\circ} \\
\text { (Back Side) }\end{array}$ & $\begin{array}{c}\boldsymbol{\theta}=\mathbf{0}^{\circ} \\
\text { (Front Side) }\end{array}$ & $\begin{array}{c}\theta=\mathbf{1 8 0}^{\circ} \\
\text { (Back Side) }\end{array}$ \\
\hline & $\begin{array}{l}\text { PANEL } \\
\text { NO }\end{array}$ & $\begin{array}{c}\text { Force } \\
\mathbf{F}(-\mathrm{Z}) \mathbf{N}\end{array}$ & $\begin{array}{c}\text { Force } \\
\mathbf{F}(\mathbf{Z}) \mathbf{N} \\
\end{array}$ & $\begin{array}{c}\text { Force } \\
\text { F }(-Z) \mathbf{N} \\
\end{array}$ & $\begin{array}{l}\text { Force } \\
\text { F(Z)N }\end{array}$ \\
\hline \multirow{7}{*}{ ROW-1 } & PANEL 1 & 2151.7 & 1241.45 & 1445.62 & 473.725 \\
\hline & PANEL 2 & 2092.88 & 1265.63 & 1479.42 & 530.815 \\
\hline & PANEL 3 & 2205.83 & 1223.93 & 1434.23 & 455.965 \\
\hline & PANEL 4 & 2170.74 & 1252.8 & 1431.33 & 473.4 \\
\hline & PANEL 5 & 2080.44 & 1240.4 & 1508.07 & 462.2 \\
\hline & PANEL 6 & 2147.52 & 1246.39 & 1464.62 & 487.195 \\
\hline & PANEL 7 & 2145.79 & 1256.22 & 1460.66 & 490.11 \\
\hline \multirow{7}{*}{ ROW-2 } & PANEL 8 & 1137.92 & 1131.27 & 547.23 & 409.635 \\
\hline & PANEL 9 & 1183.56 & 1034.92 & 576.83 & 334.46 \\
\hline & PANEL 10 & 1100.00 & 1056.62 & 571.62 & 1184.23 \\
\hline & PANEL 11 & 1222.66 & 1105.45 & 572.47 & 1174.54 \\
\hline & PANEL 12 & 1156.16 & 1090.02 & 591.85 & 1188.53 \\
\hline & PANEL 13 & 1220.54 & 1091.31 & 579.86 & 1153.46 \\
\hline & PANEL 14 & 1209.21 & 1052.99 & 567.55 & 963.42 \\
\hline \multirow{7}{*}{ ROW-3 } & PANEL 15 & 1189.67 & 914.7 & 526.35 & 675.15 \\
\hline & PANEL 16 & 1214.3 & 907.45 & 493.62 & 766.46 \\
\hline & PANEL 17 & 1158.3 & 899.13 & 514.42 & 793.23 \\
\hline & PANEL 18 & 1173.99 & 941.01 & 524.67 & 815.78 \\
\hline & PANEL 19 & 1207.59 & 869.18 & 496.94 & 810.27 \\
\hline & PANEL 20 & 1150.35 & 882.12 & 522.16 & 698.81 \\
\hline & PANEL 21 & 1190.51 & 972.86 & 522.16 & 698.81 \\
\hline \multirow{7}{*}{ ROW-4 } & PANEL 22 & 1020.93 & 2043.24 & 512.3 & 1386.63 \\
\hline & PANEL 23 & 1100.19 & 2097.22 & 523.16 & 1430.75 \\
\hline & PANEL 24 & 1090.38 & 2066.68 & 546.59 & 1431.64 \\
\hline & PANEL 25 & 1070.18 & 2035.38 & 531.33 & 1416.71 \\
\hline & PANEL 26 & 1150.17 & 2139.29 & 512.65 & 1412.66 \\
\hline & PANEL 27 & 1100.54 & 2065.4 & 527.74 & 1422.64 \\
\hline & PANEL 28 & 1080.96 & 2065.16 & 530.07 & 1393.46 \\
\hline
\end{tabular}


Table.7 Comparing the Lift forces between Rectangular and Hexagonal solar panels at wind velocity $5 \mathrm{~m} / \mathrm{s}$ mounted at ground

\begin{tabular}{|c|c|c|c|c|c|}
\hline \multicolumn{6}{|c|}{ Comparing of wind effects on solar farm mounted at ground at Velocity $5 \mathrm{~m} / \mathrm{s}$} \\
\hline \multicolumn{2}{|c|}{ Shape of the panel } & \multicolumn{2}{|c|}{$\begin{array}{l}\text { Rectangular Solar } \\
\text { Panels }\end{array}$} & \multicolumn{2}{|c|}{$\begin{array}{c}\text { Optimized Hexagonal solar } \\
\text { panels }\end{array}$} \\
\hline \multirow[b]{2}{*}{ ROWS } & $\begin{array}{l}\text { Wind } \\
\text { Angle }\end{array}$ & $\begin{array}{c}\boldsymbol{\Theta}=\mathbf{0}^{\circ} \\
\text { (Front Side) }\end{array}$ & $\begin{array}{c}\begin{array}{c}\theta=\mathbf{1 8 0}^{\circ} \\
\text { (Back Side) }\end{array} \\
\end{array}$ & $\begin{array}{c}\boldsymbol{\theta}=\mathbf{0}^{\mathbf{o}} \\
\text { (Front Side) }\end{array}$ & 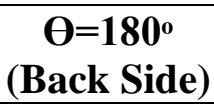 \\
\hline & $\begin{array}{l}\text { PANEL } \\
\text { NO }\end{array}$ & $\begin{array}{c}\text { Force } \\
\text { F(Y) N }\end{array}$ & $\begin{array}{l}\text { Force } \\
\text { F(Y) N }\end{array}$ & $\begin{array}{l}\text { Force } \\
\text { F(Y) N }\end{array}$ & $\begin{array}{l}\text { Force } \\
\text { F(Y)N }\end{array}$ \\
\hline \multirow{7}{*}{ ROW-1 } & PANEL 1 & 551.29 & 555.927 & 173.54 & 138.53 \\
\hline & PANEL 2 & 557.71 & 555.67 & 178.89 & 159.64 \\
\hline & PANEL 3 & 551.68 & 551.02 & 176.25 & 163.19 \\
\hline & PANEL 4 & 557.31 & 557.54 & 170.94 & 163.86 \\
\hline & PANEL 5 & 552.79 & 552.38 & 182.64 & 163.77 \\
\hline & PANEL 6 & 556.13 & 555.98 & 176.74 & 160.94 \\
\hline & PANEL 7 & 555.09 & 556.21 & 176.18 & 137.54 \\
\hline \multirow{7}{*}{ ROW-2 } & PANEL 8 & 555.7 & 555.86 & 58.94 & 113.92 \\
\hline & PANEL 9 & 556.54 & 556.72 & 62.89 & 143.31 \\
\hline & PANEL 10 & 556.1 & 556.08 & 64.29 & 145.77 \\
\hline & PANEL 11 & 555 & 554.86 & 62.98 & 144.46 \\
\hline & PANEL 12 & 561.58 & 561.52 & 64.85 & 147.38 \\
\hline & PANEL 13 & 559.99 & 559.81 & 64.56 & 141.36 \\
\hline & PANEL 14 & 553.25 & 553.73 & 61.63 & 115.3 \\
\hline \multirow{7}{*}{ ROW-3 } & PANEL 15 & 550.71 & 550.4 & 57.21 & 77.15 \\
\hline & PANEL 16 & 558.33 & 558.5 & 54.89 & 90.18 \\
\hline & PANEL 17 & 556.42 & 556.41 & 57.19 & 92.91 \\
\hline & PANEL 18 & 544.66 & 544.53 & 58.37 & 95.56 \\
\hline & PANEL 19 & 548.5 & 549 & 54.78 & 96.01 \\
\hline & PANEL 20 & 545.39 & 546 & 56.11 & 95.38 \\
\hline & PANEL 21 & 552.13 & 552 & 57.29 & 80.35 \\
\hline \multirow{7}{*}{ ROW-4 } & PANEL 22 & 536.17 & 536 & 56.24 & 171.03 \\
\hline & PANEL 23 & 539.91 & 540 & 59.06 & 177.38 \\
\hline & PANEL 24 & 545.75 & 546 & 59.88 & 176.39 \\
\hline & PANEL 25 & 539.25 & 539 & 58.98 & 175.03 \\
\hline & PANEL 26 & 541.95 & 542 & 56.31 & 174.52 \\
\hline & PANEL 27 & 539.45 & 539 & 58.02 & 175.63 \\
\hline & PANEL 28 & 539.96 & 540 & 57.45 & 171.04 \\
\hline
\end{tabular}

Table.8 Comparing the Lift forces between Rectangular and Hexagonal solar panels at wind velocity $25 \mathrm{~m} / \mathrm{s}$ mounted at ground

\begin{tabular}{|c|c|c|c|c|c|}
\hline \multicolumn{6}{|c|}{ Comparing of wind effects on solar farm mounted at ground at Velocity $25 \mathrm{~m} / \mathrm{s}$} \\
\hline \multicolumn{2}{|c|}{ Shape of the panel } & \multicolumn{2}{|c|}{$\begin{array}{l}\text { Rectangular Solar } \\
\text { Panels }\end{array}$} & \multicolumn{2}{|c|}{$\begin{array}{c}\text { Optimized Hexagonal solar } \\
\text { panels }\end{array}$} \\
\hline ROWS & $\begin{array}{l}\text { Wind } \\
\text { Angle }\end{array}$ & $\begin{array}{c}\boldsymbol{\theta}=0^{\circ} \\
\text { (Front Side) }\end{array}$ & $\begin{array}{c}\begin{array}{c}\Theta=\mathbf{1 8 0}^{\circ} \\
\text { (Back Side) }\end{array}\end{array}$ & $\begin{array}{c}\boldsymbol{\theta}^{=}=\mathbf{0}^{\circ} \\
\text { (Front Side) }\end{array}$ & $\begin{array}{c}\theta=\mathbf{1 8 0}^{\circ} \\
(\text { Back Side) }\end{array}$ \\
\hline
\end{tabular}




\begin{tabular}{|c|c|c|c|c|c|}
\hline & $\begin{array}{c}\text { PANEL } \\
\text { NO }\end{array}$ & $\begin{array}{c}\text { Force } \\
\text { F(Y) N }\end{array}$ & $\begin{array}{c}\text { Force } \\
\text { F(Y) N }\end{array}$ & $\begin{array}{c}\text { Force } \\
\text { F(Y) N }\end{array}$ & $\begin{array}{l}\text { Force } \\
\text { F(Y)N }\end{array}$ \\
\hline \multirow{7}{*}{ ROW-1 } & PANEL 1 & 6555.39 & 3731.56 & 4370.18 & 3433.92 \\
\hline & PANEL 2 & 6378.60 & 3794.7 & 4480.54 & 3977.03 \\
\hline & PANEL 3 & 6662.22 & 3658.96 & 4300.25 & 4065.00 \\
\hline & PANEL 4 & 6592.41 & 3733.14 & 4310.16 & 4074.75 \\
\hline & PANEL 5 & 6386.31 & 3718.69 & 4570.47 & 4074.37 \\
\hline & PANEL 6 & 6468.91 & 3716.81 & 4400.64 & 4010.83 \\
\hline & PANEL 7 & 6358.52 & 3768.66 & 4410.72 & 3421.63 \\
\hline \multirow{7}{*}{ ROW-2 } & PANEL 8 & 3405.12 & 3383.26 & 1470.31 & 2823.71 \\
\hline & PANEL 9 & 3539.01 & 3089.72 & 1550.39 & 3566.30 \\
\hline & PANEL 10 & 3224.07 & 3119.11 & 1560.29 & 3639.81 \\
\hline & PANEL 11 & 3661.71 & 3302.57 & 1520.41 & 3610.66 \\
\hline & PANEL 12 & 3460.39 & 3265.81 & 1610.07 & 3658.00 \\
\hline & PANEL 13 & 3620.80 & 3243.09 & 1580.91 & 3532.51 \\
\hline & PANEL 14 & 3578.53 & 3127.6 & 1530.78 & 2864.02 \\
\hline \multirow{7}{*}{ ROW-3 } & PANEL 15 & 3549.41 & 2742.35 & 1420.06 & 1912.43 \\
\hline & PANEL 16 & 3547.94 & 2724.02 & 1340.59 & 2238.60 \\
\hline & PANEL 17 & 3509.77 & 2705.41 & 1410.14 & 2329.48 \\
\hline & PANEL 18 & 3446.03 & 2817.74 & 1440.06 & 2391.00 \\
\hline & PANEL 19 & 3605.53 & 2602.59 & 1360.15 & 2379.8 \\
\hline & PANEL 20 & 3417.22 & 2642.39 & 1390.05 & 2315.62 \\
\hline & PANEL 21 & 3545.22 & 2925.33 & 1420.74 & 1994.85 \\
\hline \multirow{7}{*}{ ROW-4 } & PANEL 22 & 3068.8 & 6210.82 & 1380.66 & 4277.63 \\
\hline & PANEL 23 & 3273.29 & 6415.11 & 1440.21 & 4435.55 \\
\hline & PANEL 24 & 3310.28 & 6267.61 & 1510.58 & 4417.85 \\
\hline & PANEL 25 & 3197.51 & 6220.87 & 1470.94 & 4375.21 \\
\hline & PANEL 26 & 3402.20 & 6473.71 & 1400.32 & 4317.69 \\
\hline & PANEL 27 & 3269.66 & 6255.42 & 1450.16 & 4386.89 \\
\hline & PANEL 28 & 3225.86 & 6316.42 & 1430.92 & 4289.59 \\
\hline
\end{tabular}

Here we are comparing the drag and lift forces for ground mounted rectangular and hexagonal solar panels at velocities $5 \mathrm{~m} / \mathrm{s}$ and $25 \mathrm{~m} / \mathrm{s}$. Optimized solar panels are the futuristic panels with respect to efficiency and with standing for the wind at all locations. The above tables clearly say that hexagonal panels are the best panels compared with the rectangular panel.

\section{Conclusion}

By using Computational Fluid Dynamics, the present work examines the effects of the wind loads on the Solar panels. Multiple scenarios are simulated to evaluate the forces on the behavior of loads on ground mounted solar farms and roof mounted solar panels. A 28 panel array of ground mounted solar farm is simulated for the conditions of 5 and $25 \mathrm{~m} / \mathrm{s}$ speeds from front and behind. Data obtained shows that in the ground mounted scenario for $5 \mathrm{~m} / \mathrm{s} \mathrm{\&}$ $25 \mathrm{~m} / \mathrm{s}$, maximum forces occurred from panel 1 to panel 7 on front face of the solar panel. Based on the results obtained, it is noted that for traditional shaped solar panels the pressure distribution on the front face of the solar panels is very high. Thus there is a need to design optimized solar panel shape. An array of 28 panels is simulated in full scale conditions. The 
forces are reduced on this model when compared with the traditional model at maximum velocity. The maximum force occurred on panel 1 to panel 7 for traditional model is $6500 \mathrm{~N}$ and the forces occurred on the optimized solar panel is $4100 \mathrm{~N}$ in case of $25 \mathrm{~m} / \mathrm{s}$ speed when the direction of wind is normal to the panel. It is recommended that for rigorous flow scenarios, the optimized solar panel design is adequately suitable in cyclone prone areas and shore regions.. A 3 Panel array of roof mounted solar panel is simulated considering active roof concept. For $5 \mathrm{~m} / \mathrm{s}$ and $25 \mathrm{~m} / \mathrm{s}$ front and behind flow is simulated. For $25 \mathrm{~m} / \mathrm{s}$ the maximum load is occurring on Panel 1 with $1100 \mathrm{~N}$ force for traditional solar panels and a force of $925 \mathrm{~N}$ is occurring on Panel 2 for optimized solar Panel. Observation of the behavior of loads on roof mounted solar panels with traditional and optimized panel design is also recommended. This scenario is considered to investigate the suitability of optimized solar panel in urban environment.

\section{Acknowledgement}

Authors PSR, KS wish to acknowledge the Department of Science and Technology for providing Funding through DST-FIST Level-1 Scheme to Department of Physics, KLEF; File No: SR/FST/PS-1/2018/35 and K. L. E. F. for their support during the work.

\section{References:}

(1) Robert N. Meroney and David E. Neff: Wind effects on roof-mounted solar photovoltaic arrays: CFD and wind-tunnel evaluation. The Fifth International Symposium on Computational Wind Engineering (CWE2010) Chapel Hill, North Carolina, USA May 23- 27, 2010.

(2) Girma T. Bitsuamlak, Agerneh K. Dagnew, James Erwin: Evaluation of wind loads on solar panel modules using CFD, The Fifth International Symposium on Computational Wind Engineering (CWE2010), Chapel Hill, North Carolina, USA May 23-27, 2010

(3) Jubayer C. M., Hangan H: A numerical approach to the investigation of wind loading on an array of ground mounted solar photovoltaic (PV) panels. Journal of Wind Engineering and Industrial Aerodynamics, 153. (2016) 60-70.

(4) Warsido W. P., Bitsuamlak G. T., Barata J., Gan Chowdhury A.: Influence of spacing parameters on the wind loading of solar array. Journal of Fluids and Structures, 48. (2014) 295-315.

(5) Cain J. H., Banks D: Wind Loads on Utility Scale Solar PV Power Plants. 2015 SEAOC Convention Proceedings. 1-8.

(6) Alluri S. K. R, Shit T., Gujjula D., Phani Kumar S. V. S., Ramana Murthy M. V.: Feasibility study on fixed platforms for offshore wind turbine in India. Journal of Structural Engineering (India), 41/1. (2014) 12481256.

(7) Victor Udoewa and Vinod Kumar: Computational Fluid Dynamics, In book: Applied Computational Fluid Dynamics, Chapter · March 2012

(8) P. Surendra Reddy, Swapna Koneru, G. Kiran Kumar , Numerical Investigation of Wind load effect on optimized solar Panel design at high Altitudes,Test Engineering and Management, march 2020,vol 83, page 21752-21764.

(9) P.Surendra Reddy, *G. Kiran Kumar, Swapna koneru,3, Numerical Investigation and Optimization of Aerodynamic Effect on Solar Panels at High Altitudes in Urban Environment, International Journal of control and automation, vol. 13, no.2. (2020), pp. 428- 439.

(10) G.SuryaNarayana, Kamakshaiah Kolli, Fuzzy K-means clustering with fast density peak clustering on multivariate kernel estimator with evolutionary multimodal optimization clusters on a large dataset, Multimedia Tools and Applications Vol. 80,Issue No.3(2021), pp. 4769-4787,ISSN: 1380-7501, DOI 10.1007/s11042-020-09718-4.

(11) Kolli Kamakshaiah, Dr.R.Seshadri,Prototype survey analysis of different information retrieval classification and grouping approaches for categorical information," 2017 International Conference on Intelligent Computing and Control (I2C2), Coimbatore, 2017, pp. 1-7, Doi: 10.1109/I2C2.2017.8321825, Part of ISBN: 9781538603741. 